EESTI NSV TEADUSTE AKADEEMIA TOIMETISED. XI KOIDE

FOOSIKALIS-MATEMAATILISTE JA TEHNILISTE TEADUSTE SEERIA. 1962, NR. 1

ИЗВЕСТИЯ АКАДЕМИИ НАУК ЭСТОНСКОН ССР. ТОМ ХI

СЕРИЯ ФИЗИКО-МАТЕМАТИЧЕСКИХ И ТЕХНИЧЕСКИХ НАУК, 1962, N 1

\title{
О ВОЗМОЖНОСТИ УЛУЧШЕНИЯ МЕТОДА РАЗЛИЧЕНИЯ СЛАНЦА ОТ ИЗВЕСТНЯКА ПРИ ПОМОЩИ ДВУХ ПУЧКОВ ГАММА-ИЗЛУЧЕНИЯ РАЗНОЙ ПРОНИЦАЕМОСТИ
}

\section{и. БРАУН}

Как было показано в работе [2], при помощи двух пучков гамма-излучения раз. ной проницаемости можно различить друг от друга материалы с разными химическими составами независимо от линейных размеров их кусков. При этом, однако, для переработки информации, полученной от радиоактивных счетчиков, требуется довольно сложная электронная аппаратура (вычислительная машина непрерывного действия). Дальнейшие исследования показали, что если изменить схемы переработки информации, то можно добиться упрощения аппаратуры, хотя при этом уменьшается и диапазон изменения размеров различаемых кусков.

В данной работе рассматривается возможность расширения диапазона изменения размеров кусков, в котором удастся однозначно различить материалы при использовании двух гамма-излучений и упрощенной схемы переработки информации.

\section{Теоретические основы}

Идея предлагаемого метода заключается в том, что один пучок гамма-излучения используется в качестве «толщиномера» кусков материала и другой - «сортировщика» материалов.

Если обозначнть: $I_{0}^{1}$ и $I_{0}^{2}-$ интенсивность соответственно первого и второго пучков гамма-нзлучения перед поглотителем;

$\mu_{\mathrm{r}}, \mu_{\mathrm{a}}-$ линейные коэффициенты ослабления интенсивности излучения при энергин гамма-квантов $E_{1}$;

$\delta_{r}, \delta_{a}-$ линейные коэффициенты ослабления интенсивности излучения при энергии гамма-квантов $E_{2} ; x$ - толщина кусков,

то интенсивность этих двух пучков гамма-излучения после прохождения толщины $x$ будет:

а) в обогащаемом материале

$$
\begin{aligned}
& I_{2}^{1}=I_{0}^{1} e^{-\mu_{\mathrm{r}} x} \\
& I_{\mathrm{r}}^{2}=I_{0}^{2} e^{-\hat{\phi}_{\mathrm{r}} x}
\end{aligned}
$$

б) в породе

$$
\begin{aligned}
& I_{\mathrm{a}}^{1}=I_{0}^{1} e^{-\mu_{\mathrm{a}} x} \\
& I_{\mathrm{a}}^{2}=I_{0}^{2} e^{-\delta_{\mathrm{a}} x}
\end{aligned}
$$


В дальнейшем будем данный кусок сравнивать с куском другого материала н требовать, чтобы интенсивность первого пучка изменялась одинаково при прохождении обоих кусков, т. е., чтобы

$$
I_{\mathrm{r}}^{1}=I_{\mathrm{a}}^{1}=I_{0}^{1} e^{-\mu_{\mathrm{r}} x}=I_{0}^{1} e^{-\mu_{\mathrm{a}} x^{\prime}} .
$$

Отсюда получаем соотношение между размерами кусков породы и обогащаемого материала

$$
x^{\prime}=\frac{\mu_{\mathrm{r}}}{\mu_{\mathrm{a}}} x
$$

Тогда при прохождении этих же кусков пучком II получаются разные интенсивности, по разности которых можно различить материалы. В этом случае

$$
\frac{\mu_{\mathrm{r}}}{\mu_{\mathrm{a}}}-\frac{\delta_{\mathrm{r}}}{\delta_{\mathrm{a}}} \neq 0 .
$$

При выполнении условия (5) относительная разность интенсивностей прошедших излучений при облучении пучком II обогащаемого материала и породы выражается следующим образом:

$$
G=\frac{1}{I_{0}^{2}}\left(I_{\mathrm{r}}^{2}-I_{\mathrm{a}}^{2}\right)=e^{-{ }_{\mathrm{r}} x}-e^{-\delta_{\mathrm{a}} \frac{\mu_{\mathrm{r}}}{\mu_{\mathrm{a}}} x},
$$

где $x$ - размеры кусков обогащаемого материала.

Из уравнения (8) видно, что если значения линейных коэффициентов ослабления излучения изменяются в зависимости от энергии гамма-квантов одинаково, т. е. $\delta_{\mathrm{a}}=\mu_{\mathrm{a}}$ и $\delta_{\mathrm{r}}=\mu_{\mathrm{r}}$, то $G$ равняется нулю и различить материалы друг от друга по химичесқому составу невозможно. Но чем больше левая часть неравенства (7) отличается от нуля, тем более высокие значения имеет $G$ и тем легче различить материалы друг от друга. Для того, чтобы левая часть неравенства (7) как можно больше отличалась от нуля, целесообразно выбрать энергию гамма-квантов $\left(E_{1}\right)$ пучка I в области энергии, где ослабление интенсивности пучка обусловлено в основном комптоновскнм рассеянием, и энергию гамма-квантов $\left(E_{2}\right)$ пучка II в области, где ослабление интенсивности излучения обусловлено фотопоглощением.

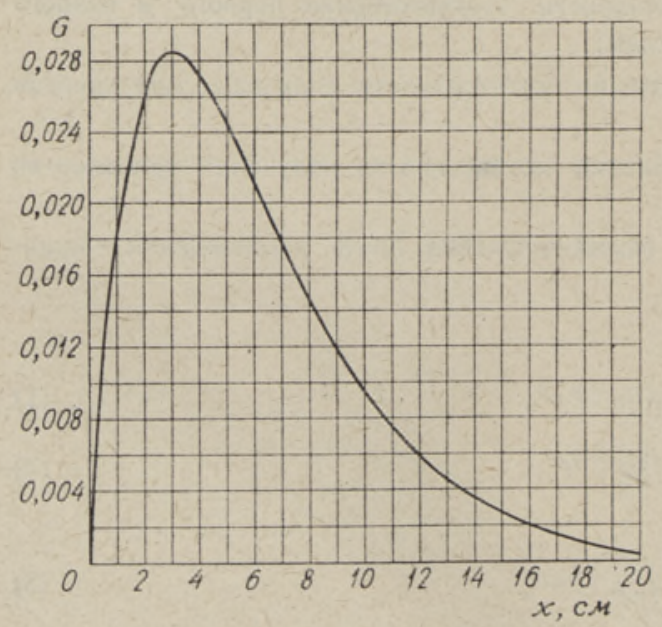

Фиг. 1. Зависимость функции $G$ от толщины кусков $x$.
На фиг. 1 уравнение (8) пред ставлено графически. Из графика видно, что при увеличении размеров кусков величина $G$ быстро достигает своего максимального значения и затем, при дальнейшем увеличении размеров кусков, спадает асимптотическь до нуля. Размеры кусков $x_{\max }$, прн которых $G$ достигает максимального значения, можно найти из условия *

$$
\left.\frac{d G}{d x}\right|_{x=x_{\max }}=0
$$

* Размеры кусков породы в $\mu_{\mathrm{a}} / \mu_{\mathrm{r}}$ раз меньше размеров кусков обогащаемого материала [см. формулу (6)]. 
Тогда

$$
x_{\max }=\frac{\ln \left(\frac{{ }_{\mathrm{r}}}{{ }_{\delta_{\mathrm{a}}}} \frac{\mu_{\mathrm{a}}}{\mu_{\mathrm{r}}}\right)}{\delta_{\mathrm{r}}-\delta_{\mathrm{a}} \frac{\mu_{\mathrm{r}}}{\mu_{\mathrm{a}}}} .
$$

Заменив в уравнении (8) $x$ через $x_{\max }$, получим максимальное значение функции $G$

$$
G_{\max }=\exp \left(-\frac{\ln \left(\frac{\delta_{\mathrm{r}}}{\delta_{\mathrm{a}}} \frac{\mu_{\mathrm{a}}}{\mu_{\mathrm{r}}}\right)}{1-\frac{\delta_{\mathrm{a}}}{\delta_{\mathrm{r}}} \frac{\mu_{\mathrm{r}}}{\mu_{\mathrm{a}}}}\right)-\exp \left(-\frac{\ln \left(\frac{\delta_{\mathrm{r}}}{\delta_{\mathrm{a}}} \frac{\mu_{\mathrm{a}}}{\mu_{\mathrm{r}}}\right)}{\frac{{ }_{\mathrm{r}}}{\delta_{\mathrm{a}}} \frac{\mu_{\mathrm{a}}}{\mu_{\mathrm{r}}}-1}\right) .
$$

Обозначая

$$
\frac{\delta_{\mathrm{r}}}{\delta_{\mathrm{a}}}=\gamma_{\mathrm{II}}, \frac{\mu_{\mathrm{r}}}{\mu_{\mathrm{a}}}=\gamma_{\mathrm{I}}, \gamma_{\mathrm{I}}-\gamma_{\mathrm{II}}=\sigma
$$

и подставляя соответствующие величины в уравнение (10), получаем

$$
G_{\max }=\left(1-\frac{\sigma}{\gamma_{1}}\right)^{\frac{\gamma_{1}}{\sigma}-1}-\left(1-\frac{\sigma}{\gamma_{1}}\right)^{\frac{\gamma_{I}}{\sigma}} .
$$

Переменная $\sigma / \gamma_{1}$ характеризует взаимодействие между веществом и излучением. Она вполне определена свойствами контролируемого материала и пучков излучения I и II. В сущности, $\sigma / \gamma_{\text {I }}$ обозначает относительную разницу между отношениями линейных коэффициентов ослабления для разных гамма-квантов. Обозначив ее через $\varepsilon$, получаем уравнение (12) в виде

$$
G_{\max }=\varepsilon(1-\varepsilon)^{\frac{1-\varepsilon}{\varepsilon}} .
$$

Из фиг. 2, представляющей уравнение (13), видно, что кривая $G_{\max }$ в промежутке $0<\varepsilon<0,5$ довольно близка к прямой. Следовательно, для получения больших значений $G_{\max }$ ни одно из значений $\boldsymbol{\varepsilon}$ особых преимуществ не имеет: чем выше значение $\varepsilon$, тем более высокие значения имеет $G_{\max }$.

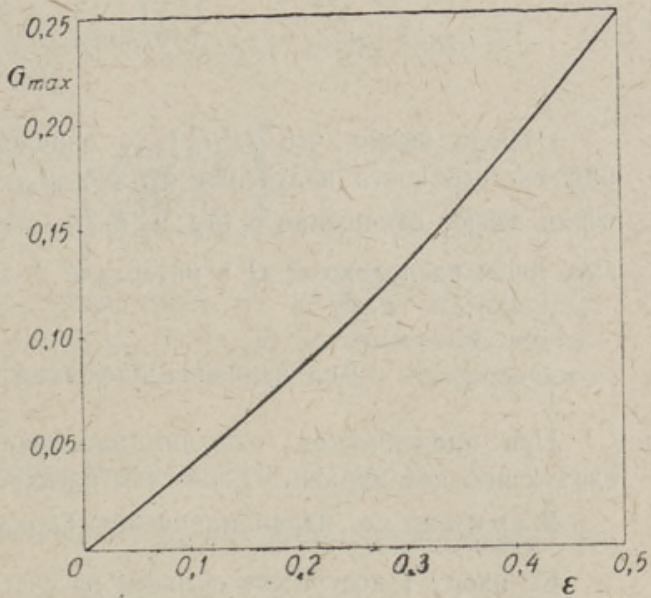

Фиг. 2. Зависимость функции $G_{\max }$ от $\varepsilon$.

Используя обозначения (11), получаем уравнение (9) в следующем виде:

$$
x_{\max }=-\frac{1-\varepsilon}{\delta_{\mathrm{r}} \varepsilon} \ln (1-\varepsilon) .
$$

Отсюда видно: чем меньше по величине $\delta_{r}$, тем больше $x_{\max }$. Это означает, что энергия гамма-квантов «пучка-сортировщика» при облучении больших кусков контролируемого материала не может быть чересчур малой. В противном случае $G_{\max }$ будет лежать в области слишком малых размеров кусков и при облучении кусков крупного размера сигнал $G$ будет слишком мал, чтобы его удовлетворительно зарегистрировать. 
Поскольку размеры кусков «измеряются» при помощи пучка I, то целесообразно в (8) заменить $x$ на $I^{1}$. Из уравнения (1) получаем

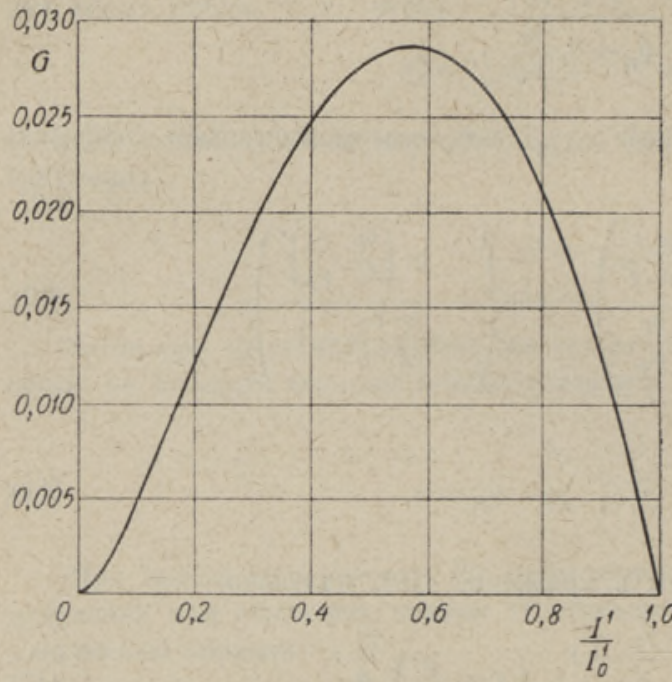

Фиг. 3. Зависимость функции $G$ от относительной интенсивности $I^{1} / I_{0}^{1}$ пучка I.

$$
x=\frac{\ln I_{0}^{1}-\ln I^{1}}{\mu_{\Gamma}} .
$$

Тогда уравнение (8) примет следующий вид:

$$
G=\left(\frac{I^{1}}{I_{0}^{1}}\right)^{\frac{\delta_{\mathrm{r}}}{\mu_{\mathrm{r}}}}-\left(\frac{I^{1}}{I_{0}^{1}}\right)^{\frac{\delta_{\mathrm{a}}}{\mu_{\mathrm{a}}}} .
$$

На фиг. 3 зависимость между $G$ и $I^{1} / I_{0}^{1}$ представлена графически. Из рисунка видно, что функция $G$ имеет максимальную величину при некотором значении $I^{1} / I_{0}^{1}$; обозначим ее через $\left(I^{1} / I_{0}^{1}\right)_{\max }$. Уравнение для определения этой величины получим из (14), заменив в нем $x_{\max }$ выражением $x$ из уравнения (15)

$$
\left(\frac{I^{1}}{I_{0}^{1}}\right)_{\max }=\frac{1}{(1-\varepsilon)^{\frac{\mu_{r}}{\partial_{\mathrm{r}}} \frac{1-\varepsilon}{\varepsilon}}} .
$$

Отсюда видно, что $\left(I^{1} / I_{0}^{1}\right)_{\max }$ определено значениями коэффициентов ослабления интенсивности излучения $\delta_{\mathrm{a}}, \delta_{\mathrm{r}}, \mu_{\mathrm{a}}, \mu_{\mathrm{r}}$. Но при этом чем более высокие значения имеют отношения $\delta_{\mathrm{a}} / \mu_{\mathrm{a}}$ и $\delta_{\mathrm{r}} / \mu_{\mathrm{r}}$, тем более несимметрично относительно краевых точек расположено $G$ в интервале $0<I^{1} / I_{0}^{1}<1$.

\section{Два варианта блок-схем электронной аппаратуры}

При практическом решенин рассматриваемого вопроса целесообразно изучить следующие два варианта блок-схем электронной аппаратуры.

В арнант а. Принципиальная блок-схема представлена на фиг. 4.

Ко входу I поступают сигналы из счетчика, регистрирующего излучения пучка I, и к входу II - из счетчика, регистрирующего излучения пучка II.

Фиг. 4. Блок-схема вычнслительного устройства (вариант а).

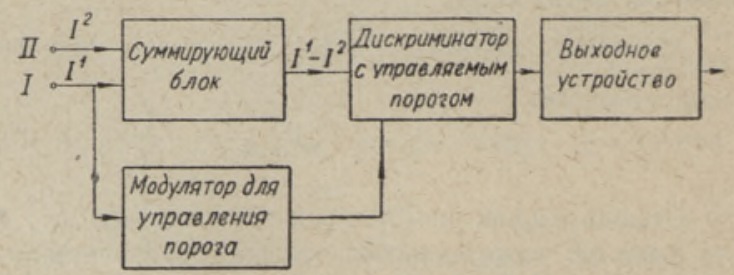

Если величнна сигнала, поступающего ко входу I, не изменяется, то величина сигналов канала II зависит от химического состава контролируемых материалов. В этом случае разница между величинами сигналов обогащаемого материала и породы пропорциональна значениям функции $G$ [формула (16)]. 
Допустим, что дискриминатор имеет постоянный уровень порога дискриминации. В этом случае возможность различить сигналы обогащаемого материала от сигналов породы ограничена небольшим интервалом размеров кусков [']. Диапазон различения расширяется, если величина порогового напряжения изменяется соответственно изменениям размера кусков. Очевидно, кривая изменения величины порогового напряжения дискриминации должна находиться между кривыми обогащаемого мақериала и породы, которые можно выразить, если заменить $x$ в уравнениях (2) и (3) через I! (15), следующим образом:

$$
\begin{aligned}
& I_{\mathrm{r}}^{2}=I_{0}^{2}\left(\frac{I^{1}}{I_{0}^{1}}\right)^{\frac{\delta_{\mathrm{r}}}{\mu_{\mathrm{r}}}} \\
& I_{\mathrm{a}}^{2}=I_{0}^{2}\left(\frac{I^{1}}{I_{0}^{1}}\right)^{\frac{\delta_{\mathrm{a}}}{\mu_{\mathrm{a}}}} .
\end{aligned}
$$

Следователько, при «плохом» обогащаемом материале (близким по свойствам к породе) и при «плохой» породе (близкой по свойствам к обогащаемому материалу) кривая выходного сигнала модулятора должна находиться между кривыми $I_{\mathrm{r}}^{2}$ и $I_{\mathrm{a}}^{2}$, на равных дистанциях от этих кривых. Очевидно, уравнение модулятора выразится в виде

$$
U_{\text {вых }}=a\left(\frac{I_{\mathrm{a}}^{2}}{I_{0}^{2}}+\frac{1}{2} G\right) .
$$

Заменяя $I^{1} / I_{0}^{1}$ нерез $U_{\mathrm{Bx}} / U_{\mathrm{Bx}}^{\max }$, получаем после некоторых преобразованнй

$$
U_{\mathrm{Bdx}}=\frac{a}{2}\left[\left(\frac{U_{\mathrm{Bx}}}{U_{\mathrm{BX}}^{\max }}\right)^{\frac{\hat{\sigma}_{\mathrm{a}}}{\mathrm{\mu}_{\mathrm{a}}}}+\left(\frac{U_{\mathrm{Bx}}}{U_{\mathrm{BX}}^{\max }}\right)^{\frac{\hat{\sigma}_{\mathrm{r}}}{\mu_{\mathrm{r}}}}\right],
$$

где $\alpha$ - коэффициент пропорциональности, зависящий от свойств аппаратуры; $U_{\text {вх }}$ - величина входного сигнала (канал I); $\delta_{\mathrm{a}} / \mu_{\mathrm{a}}$ и $\delta_{\mathrm{r}} / \mu_{\mathrm{r}}$ - отношенне лннейных козфффициентов соответственно «плохой» породы н кплохого» обогащаемого материала.

В ари ант 6. Принципиальная блок-схема представлена на фиг. 5.

K входам I и II сигналы поступают аналогично тому, как представлено на фиг. 4.

Фиг. 5. Блок-схема вычнслительного устройства (вариант б).

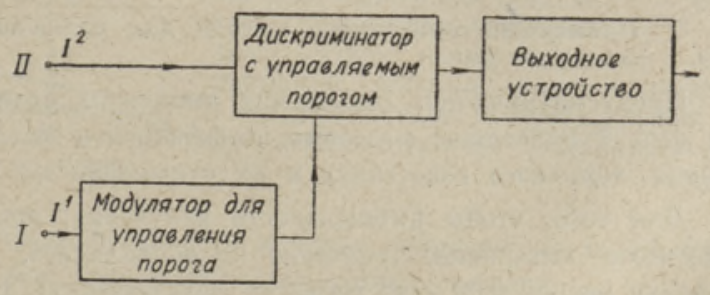

Из суммирующего блока к выходу дискриминатора поступает разность величнн сигналов каналов I и II, которая пропорциональна разности интенсивностей излучений пучков I и II. Разность интенсивностей излучений пучков I и II в зависимости от размеров кусков выражается в виде уравнения следующего тнпа [2]:

$$
\Delta I=I^{1}-I^{2}=I_{0}^{1} e^{-\mu x}-I_{0}^{2} e^{-\delta x} .
$$


Если величина сигнала в канале I не изменяется, величина сигнала в канале II зависит от химических свойств контролируемого материала. В этом случае разность сигналов при обогащаемом материале и породе пропорциональна значениям функцин $G$ [формула (16)]. Величина порогового напряжения дискриминации должна измениться в зависимости от величин сигналов в канале I, в противном случае материалы можно различить друг от друга только в некотором небольшом интервале размеров кусков [2]. Очевидно, кривая зависимости выходного сигнала модулятора от размеров кусков находится между кривыми $\Delta I_{\mathrm{a}}$ и $\Delta I_{\mathrm{r}}$. Если заменить $x$ через $I^{1}$, то $\Delta I_{\text {а }}$ и $\Delta I_{\mathrm{r}}$ выразятся следующим образом:

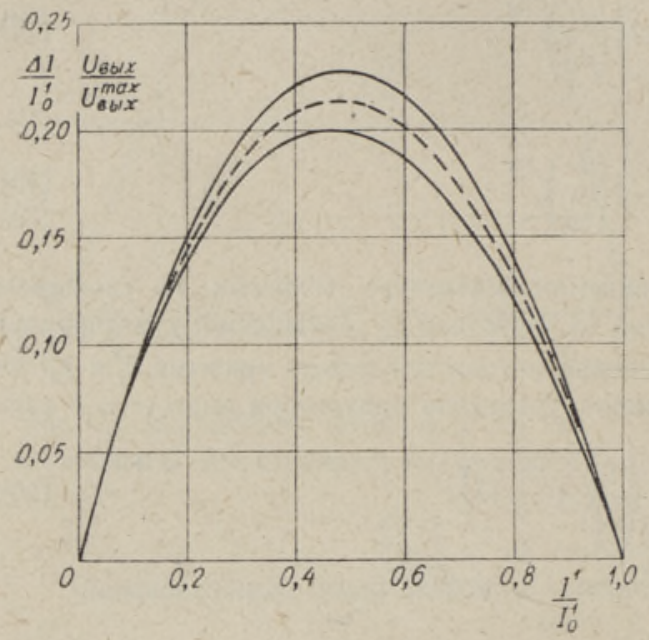

$$
\begin{aligned}
& \frac{\Delta I_{\mathrm{a}}}{I_{0}^{1}}=\frac{I^{1}}{I_{0}^{1}}-\frac{I_{0}^{2}}{I_{0}^{1}}\left(\frac{I^{1}}{I_{0}^{1}}\right)^{\frac{\delta_{\mathrm{a}}}{\Gamma_{\mathrm{a}}}}, \\
& \frac{\Delta I_{\mathrm{r}}}{I_{0}^{1}}=\frac{I^{1}}{I_{0}^{1}}-\frac{I_{0}^{2}}{I_{0}^{1}}\left(\frac{I^{1}}{I_{0}^{1}}\right)^{\frac{\sigma_{\mathrm{r}}}{\tilde{I}_{\mathrm{r}}}} .
\end{aligned}
$$

Фиг. 6. Зависимость функций $\Delta I_{\mathrm{r}} / I_{0}^{1}$, $\Delta I_{\mathrm{a}} / I_{0}^{1}$ и выходного сигнала модулятора от относительной интенсивности $I^{1} / I_{0}^{1}$ пучка I.

Кривые $\Delta I_{\mathrm{r}}$ и $\Delta I_{\mathrm{a}}$ представлены графически на фиг. 6 непрерывными линиями, а кривая изменения выходного сигнала - прерывающейся линией. Если заменить $I^{1} / I_{0}^{1}$ через $U_{\mathrm{Bx}} / U_{\mathrm{Bx}}^{\max }$, то уравнение для модулятора будет иметь следующий вид:

$$
U_{\mathrm{Bbx}}=\beta\left[\frac{U_{\mathrm{Bx}}}{U_{\mathrm{BX}}^{\max }}-\left(\frac{U_{\mathrm{Bx}}}{U_{\mathrm{BX}}^{\max }}\right)^{\frac{\delta_{\mathrm{a}}}{\mu_{\mathrm{a}}}+\frac{{ }^{\delta_{\mathrm{r}}}}{\mu_{\mathrm{r}}}}\right],
$$

где $\beta$ - коэффициент пропорциональности, зависящий от свойств аппаратуры; $\delta_{\mathrm{a}} / \mu_{\mathrm{a}}$ и $\delta_{r} / \mu_{r}$ - отношение линейных коэффициентов ослабления соответственно «плохой» породы и «плохого» обогащаемого материала.

\section{Применение описанного метода для различения сланца от известняка}

Предусматривается различение типичного сланца от типичного известняка []]. На фиг. 7 приведены массовые коэффициенты ослабления излучения для типичного сланца, типичного известняка и их отношение как функция энергии гамма-квантов.

Для того, чтобы интенсивность излучения, прошедшего большие куски контролируемого материала, чрезмерно не ослаблялась, энергия гамма-квантов пучка II выбрана равной 0,08 Мэв (область фотоэффекта). В этом случае значения массового коэффициента ослабления излучения для сланца составляют 0,212 и для известняка $0,227 \mathrm{~cm}^{2} / \mathrm{r}$ и их отношение - 0,934 . Плотность типичного сланца $-1,5$ и типичного известняка $-2,2 \mathrm{r} / \mathrm{cm}^{3}$. Тогда $\gamma_{\text {II }}$ равна 0,6367 .

Эпергня гамма-квантов пучка I должна быть больше энергин гамма-квантов пучка II (область комптоновского рассеяния), и поэтому отношение массовых козффн- 
циентов ослабления излучения выбрано равным 1,01 (см. фиг. 7). В этом случае $\gamma_{\mathrm{I}}=0,6886$ и, следовательно, $\varepsilon=0,0745$.

Приведенный случай различения сланца от известняка представлен графически на фиг. 1, нз которого видно, что $G_{\max }=0,0287$ и $x_{\max }=3,0$ см.

Если бы ошибка измерения $G$ равнялась нулю, то различить сланец от нзвестняка можно было бы при любой величнне куска, т. е. от $x=0$ до $x=\infty$. Величина ошибки измерения сужает диапазон размеров кусков. Например, если ошибка измерения $G$ равна $0,5 \%$, то минимальное значение $G$, определяемое с достаточной достоверностью, будет 0,01 , и тогда из фиг. 1 видно, что различить сланец от извеетняка удастся при размерах кусков от 0,8 до 9,7 см. При меньшей ошибке измерения диапазон размеров кусков будет соответственно больше.

На фиг. 8 представлены диапазоны различения сланца от известняка, полученные с помощью следующих методо̄в: 1) метод одного пучка [ $\left.{ }^{1}\right]$, 2) метод двух пучков гамма-излучения с постоянным порогом дискрнминации $\left.\left[{ }^{2}\right], 3\right)$ метод двух пучков гамма-излучения с переменным порогом дискримннации (настоящая работа).

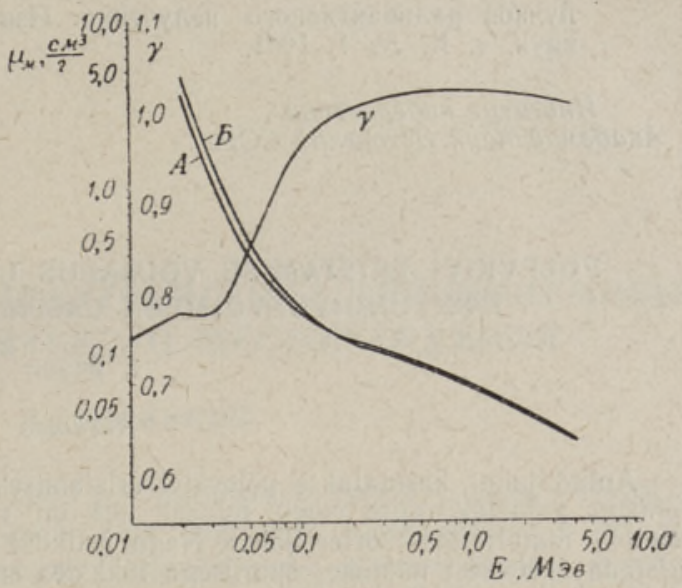

Фиг. 7. Зависимость массовых коэффициентов ослабления интенсивности излучения типичного сланца $(A)$, типичного известняка $($ (Б) и их отношения $\gamma$ от энергии гамма-квантов.

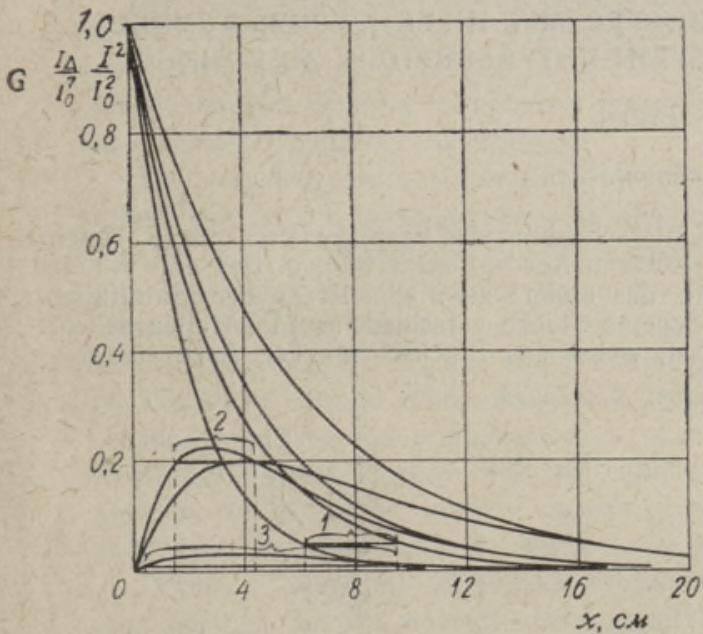

Из графика видно, что наилучшие результаты достигаются последним методом *.

Практически, т. е. при ошибке измерения $G=0,5 \%$, различение сланца от известняка піри помощи метода,

Фиг, 8. Сравнение ширины диапазонов различения сланца от известняка, полученных при помощи: 1 - одного пучка гамма-излучения; 2 двух пучков гамма-излучения с постоянным порогом дискриминацин; 3 - двух пучков гамма-излучения с переменным порогом дискриминации.

описанного в настоящей работе, можно произвести в более широком (в 6-10 раз) диапазоне изменения размеров кусков, чем с помощью других методов.

В заключение, можно заметить, что описанный метод предоставляет большие возможности для различения одного материала от другого и, очевидно, полностью удовлетворяет требованиям, предъявляемым при различении сланца от известняка.

* Прн-первых двух методах ошибка измерения предполагается равной нулю, а при третьем - $0,5 \%$. 


\section{ЛИТ Е Р А У Р А}

1. И. Бр аун, Об одной возможности использования радиоактивных изотопов для автоматизации обогащения сланца (на эстонском языке). Изв. АН ЭССР, Сер. физ.-мат. и техн. наук, т. IX, № 4, 1960.

2. И. Браун, О возможности различения сланца от известняка при помощи двух пучков радиоактивного излучения. Изв. АН ЭССР, Сер. физ.-мат. и техн. наук, т. Х, № 1, 1961.

\section{Ннститут кибернетики \\ Академии наук Эстонской ССР}

Поступила в редакцию 27. III 1961

\section{POLEVKIVI ERISTAMISE VOIMALUS LUBJAKIVIST KAHE ERINEVA LÄBITUNGIVUSVOIMEGA GAMMAKIIRGUSE VIHU ABIL}

\section{Braun}

\section{Resümee}

Antud juhul kasutatakse pōlevkivi eristamiseks lubjakivist kahte erineva neeldumisvõimega gammakiirguse vihku, millest üks on n. ö. tükkide suuruste «mõõtjaks» ja teine - materjalide «sorteerijaks». Nagu artiklist nähtub, on sellise skeemi abil vōimalik eristada üksteisest mistahes suurusega tükkides erinevaid materjale, kusjuures aparatuuri plokkskeem on vôrdlemisi lihtne. Eesti NSV Teaduste Akadeemia
Küberneetika-Instituut

Saabus toimetusse 27. III 1961

\section{EINE MÖGLICHKEIT, BRENNSCHIEFER MIT HILFE ZWEIER BONDEL GAMMASTRAHLEN VOM KALKSTEIN UNTERSCHEIDEN ZU KONNEN}

\section{Braun}

\section{Zusammenfassung}

Gegebenenfalls bedient man sich zur Unterscheidung des Brennschiefers vom Kalkstein zweier Bündel Gammastrahlen von verschiedenem Absorptionsvermögen. Das eine Bündel dient sozusagen zum «Messen» der Stücke, das andere zum «Sortieren» der Materialien. Aus dem Artikel erhellt, dass ein solches Schema Stücke verschiedener Materialien beliebiger Grösse voneinander unterscheiden lässt, wobei das Blockschema der Apparatur verhältnismässig einfach ist.

\section{Institut für Kybernetik}

der Akademie der Wissenschaften der Estnischen SSR
Eingegangen am 27. März 1961 